\title{
Stability of Reissner-Nordström Black Hole
}

\author{
G.G.L. NASHED* \\ Mathematics Department, Faculty of Science \\ Ain Shams University, Cairo, Egypt
}

(Received June 8, 2007)

\begin{abstract}
The singularity of the solutions obtained before in the teleparallel theory of gravitation is studied. Also the stability of these solutions is studied using the equations of geodesic deviation. The condition of stability is obtained. From this condition the stability of the Schwarzschild solution can be obtained.
\end{abstract}

PACS numbers: 04.20.Cv, 04.50.+h, 04.20.-q

\section{Introduction}

Static uncharged black holes in general relativity are described by the well-known Schwarzschild solution. A singularity can happen when a gravitational collapse takes place and continues until the surface of the star approaches the Schwarzschild radius, i.e., $r=2 m$ [1]. Hawking and collaborators discovered that the laws of thermodynamics have exact analogues in the properties of black holes [1-4]. As a black hole emits particles, its mass and size steadily decrease. This makes it easier to tunnel out and so the emission will continue at an ever-increasing rate until eventually the black hole radiates itself out of existence. In the long run, every black hole in the universe will evaporate in this way.

The tetrad theory of gravitation based on the geometry of absolute parallelism [5-14] can be considered as the closest alternative to general relativity, and it has a number of attractive features both from the geometrical and physical viewpoints. Absolute parallelism is naturally formulated by gauging space-time translations and underlain by the Weitzenböck geometry, which is characterized by the metricity condition and by the vanishing of the curvature tensor (constructed from the connection of the Weitzenböck geometry). Translations are closely related to the group of general coordinate transformations which underlies general

*e-mail: nashed@asunet.shams.edu.eg 
relativity. Therefore, the energy-momentum tensor represents the matter source in the field equation for the gravitational field just like in general relativity.

The tetrad formulation of gravitation was considered by Møller in connection with attempts to define the energy of gravitational field [15, 16]. For a satisfactory description of the total energy of an isolated system it is necessary that the energy-density of the gravitational field is given in terms of first- and/or second-order derivatives of the gravitational field variables. It is well known that there exists no covariant, nontrivial expression constructed out of the metric tensor. However, covariant expressions that contain a quadratic form of first-order derivatives of the tetrad field are feasible. Thus it is legitimate to conjecture that the difficulties regarding the problem of defining the gravitational energy-momentum are related to the geometrical description of the gravitational field rather than are an intrinsic drawback of the theory $[17,18]$.

In an earlier paper [19], the author used a spherically symmetric tetrad constructed by Robertson [20] to derive three different spherically symmetric space-times for the coupled gravitational and electromagnetic fields with charged source in the tetrad theory of gravitation. One of these contains an arbitrary function and generates the others. These space-times give the Reissner-Nordström metric black hole. Calculations of the energy associated with these black holes using the superpotential method given by Møller [15] have been done [19]. It has been shown that unless the time-space components of the tetrad field go to zero faster than $1 / \sqrt{r}$ at infinity, one got different results for the energy.

It is the aim of the present paper to study the singularity of the three black hole solutions obtained before [19] and then derive the condition of stability using the geodesic deviation [21]. This study is important to gain more investigation about the solutions obtained before [19]. In Sect. 2, a brief review of the three black holes is given. The singularity problem of these black holes is studied in Sect. 3. In Sect. 4, the condition of stability is given. The final section is devoted to the main results.

\section{Spherically symmetric black hole solutions}

In a previous paper the author used the teleparallel space-time in which the fundamental fields of gravitation are the parallel vector fields $b_{k}{ }^{\mu}$. In the Weitzenböck space-time the fundamental field variables describing gravity are a quadruplet of parallel vector fields $[22] b_{i}{ }^{\mu}$, which we call the tetrad field in this paper, characterized by

$$
D_{\nu} b_{i}{ }^{\mu}=\partial_{\nu} b_{i}{ }^{\mu}+\Gamma_{\lambda \nu}^{\mu} b_{i}{ }^{\lambda}=0
$$

where $\Gamma_{\lambda \nu}^{\mu}$ define the nonsymmetric affine connection coefficients. The metric tensor $g_{\mu \nu}$ is given by $g_{\mu \nu}=b^{i}{ }_{\mu} b_{i \nu}$, where summation convention is taken over $i$. Equation (1) leads to the metricity condition and the identically vanishing curvature tensor. 
The gravitational Lagrangian $L_{\mathrm{G}}$ is an invariant constructed from $g_{\mu \nu}$ and the contorsion tensor $\gamma_{\mu \nu \rho}$ given by

$$
\gamma_{\mu \nu \rho}=b_{\mu}^{i} b_{i \nu ; \rho}=\frac{1}{2}\left(T_{\nu \mu \rho}+T_{\rho \mu \nu}-T_{\mu \nu \rho}\right), \quad T_{\mu \nu \rho}=b_{\mu}^{i} b_{i \nu, \rho}-b^{i}{ }_{\nu} b_{i \mu, \rho},
$$

where the semicolon denotes covariant differentiation with respect to the Christoffel symbols and comma is the ordinary differentiation and $T_{\mu \nu \rho}$ is the torsion. It is of interest to note that the concept is as old as the gravitation theory of Einstein. The torsion notion of a variety, besides the curvature was introduced by Cartan [23] that also gave a geometric interpretation for both tensors. In teleparallel theories the gravitational interaction is described by a force similar to the Lorentz force equation of electrodynamics, with torsion playing the role of force [18].

The most general gravitational Lagrangian density invariant under parity operation is given by the form $[7,22,24]$

$$
\mathcal{L}_{\mathrm{G}}=\sqrt{-g} L_{\mathrm{G}}=\sqrt{-g}\left(\alpha_{1} \Phi^{\mu} \Phi_{\mu}+\alpha_{2} \gamma^{\mu \nu \rho} \gamma_{\mu \nu \rho}+\alpha_{3} \gamma^{\mu \nu \rho} \gamma_{\rho \nu \mu}\right)
$$

with $g=\operatorname{det}\left(g_{\mu \nu}\right)$ and $\Phi_{\mu}$ being the basic vector field defined by $\Phi_{\mu}=\gamma_{\mu \rho}^{\rho}$. Here $\alpha_{1}, \alpha_{2}$, and $\alpha_{3}$ are constants determined in such a way that the theory coincides with general relativity in the weak fields [15, 22]:

$$
\alpha_{1}=-\frac{1}{\kappa}, \quad \alpha_{2}=\frac{\lambda}{\kappa}, \quad \alpha_{3}=\frac{1}{\kappa}(1-\lambda),
$$

where $\kappa$ is the Einstein constant and $\lambda$ is a free dimensionless parameter ${ }^{\dagger}$.

The electromagnetic Lagrangian density $L_{\text {e.m. is }}[25]$

$$
L_{\mathrm{e} . \mathrm{m} .}=-\frac{1}{4} g^{\mu \rho} g^{\nu \sigma} F_{\mu \nu} F_{\rho \sigma}
$$

with $F_{\mu \nu}$ given by $F_{\mu \nu}=\partial_{\mu} A_{\nu}-\partial_{\nu} A_{\mu}$.

The gravitational and electromagnetic field equations for the system described by $L_{\mathrm{G}}+L_{\mathrm{e} . \mathrm{m}}$. are the following:

$$
G_{\mu \nu}+H_{\mu \nu}=-\kappa T_{\mu \nu}, \quad K_{\mu \nu}=0, \quad \partial_{\nu}\left(\sqrt{-g} F^{\mu \nu}\right)=0
$$

with $G_{\mu \nu}$ being the Einstein tensor of general relativity defined by

$$
G_{\mu \nu}=R_{\mu \nu}-\frac{1}{2} g_{\mu \nu} R
$$

$R_{\mu \nu}(\{\})$ is the Ricci tensor defined by

$$
R_{\mu \nu}(\{\})=\partial_{\rho}\left\{\begin{array}{l}
\rho \\
\mu \nu
\end{array}\right\}-\partial_{\nu}\left\{\begin{array}{l}
\rho \\
\mu \rho
\end{array}\right\}+\left\{\begin{array}{l}
\rho \\
\lambda \rho
\end{array}\right\}\left\{\begin{array}{l}
\lambda \\
\mu \nu
\end{array}\right\}-\left\{\begin{array}{l}
\rho \\
\lambda \nu
\end{array}\right\}\left\{\begin{array}{l}
\lambda \\
\mu \rho
\end{array}\right\}
$$

and $R(\{\})$ is the Ricci scalar $R(\{\})=g^{\mu \nu} R_{\mu \nu}$.

$H_{\mu \nu}$ and $K_{\mu \nu}$ are defined by

$$
\begin{aligned}
H_{\mu \nu} & =\lambda\left[\gamma_{\rho \sigma \mu} \gamma_{\nu}^{\rho \sigma}+\gamma_{\rho \sigma \mu} \gamma_{\nu}{ }^{\rho \sigma}+\gamma_{\rho \sigma \nu} \gamma_{\mu}{ }^{\rho \sigma}\right. \\
& \left.+g_{\mu \nu}\left(\gamma_{\rho \sigma \lambda} \gamma^{\lambda \sigma \rho}-\frac{1}{2} \gamma_{\rho \sigma \lambda} \gamma^{\rho \sigma \lambda}\right)\right]
\end{aligned}
$$

and

\footnotetext{
${ }^{\dagger}$ Throughout this paper we use the relativistic units, $c=G=1$ and $\kappa=8 \pi$.

${ }^{\ddagger}$ The Heaviside-Lorentz rationalized units will be used throughout this paper.
} 


$$
K_{\mu \nu}=\lambda\left[\Phi_{\mu, \nu}-\Phi_{\nu, \mu}-\Phi_{\rho}\left(\gamma_{\mu \nu}^{\rho}-\gamma_{\nu \mu}^{\rho}\right)+\gamma_{\mu \nu}{ }^{\rho} ; \rho\right],
$$

and they are symmetric and antisymmetric tensors, respectively. The energy-momentum tensor $T^{\mu \nu}$ is given by

$$
T^{\mu \nu}=-g_{\rho \sigma} F^{\mu \rho} F^{\nu \sigma}+\frac{1}{4} g^{\mu \nu} F^{\rho \sigma} F_{\rho \sigma} .
$$

It can be shown [22] that in the spherically symmetric case the antisymmetric part of the field equations (Eq. (6)) implies that the axial-vector part of the torsion tensor, $a_{\mu}=(1 / 3) \epsilon_{\mu \nu \rho \sigma} \gamma^{\nu \rho \sigma}$, should vanish. Then $H_{\mu \nu}$ in Eq. (8) vanishes, and the field equations (Eqs. (6)) reduce to the coupled teleparallel equivalent of the Einstein-Maxwell equations. Equations (6) then determine the tetrad field only up to local Lorentz transformations

$$
b_{\mu}^{k} \rightarrow \Lambda(x)_{\ell}^{k} b_{\mu}^{\ell}
$$

which retain the condition $a_{\mu}=0$. Hereafter we shall refer to this property of the field equations as restricted local Lorentz invariance.

The structure of the Weitzenböck spaces with spherical symmetry and three unknown functions of radial coordinate was given by Robertson [20] in the form

$$
\left(b_{i}{ }^{\mu}\right)=\left(\begin{array}{cccc}
i A & i D r & 0 & 0 \\
0 & B \sin \theta \cos \phi & \frac{B}{r} \cos \theta \cos \phi & -\frac{B \sin \phi}{r \sin \theta} \\
0 & B \sin \theta \sin \phi & \frac{B}{r} \cos \theta \sin \phi & \frac{B \cos \phi}{r \sin \theta} \\
0 & B \cos \theta & -\frac{B}{r} \sin \theta & 0
\end{array}\right),
$$

where the vector $b_{0}{ }^{\mu}$ has been taken to be imaginary in order to preserve the Lorentz signature for the metric, i.e., the functions $A$ and $D$ have to be taken as imaginary. Applying (11) to the field equations (Eqs. (6)) the author got [19] a set of non-linear partial differential equations. The solution of these equations has the following form [19]:

- first solution

$$
\begin{aligned}
& \text { if } A(R)=\frac{1}{\sqrt{1-\frac{2 m}{R}+\frac{q^{2}}{R^{2}}}}, \quad B(R)=\sqrt{1-\frac{2 m}{R}+\frac{q^{2}}{R^{2}}}, \\
& \text { and } D(R)=0, \quad \text { where } \quad R=\frac{r}{B}
\end{aligned}
$$

- second solution

$$
\text { if } \quad A=1, \quad B=1, \quad \text { and } \quad D(r)=\frac{\sqrt{2 m r-q^{2}}}{r^{2}} ;
$$

— third solution

$$
\begin{aligned}
& \text { if } A(R)=\frac{1}{\left(1-R B^{\prime}\right)}, \\
& \text { and } D(R)=\frac{1}{1-R B^{\prime}} \sqrt{\frac{2 m}{R^{3}}+\frac{q^{2}}{R^{4}}+\frac{B^{\prime}}{R}\left(R B^{\prime}-2\right)} .
\end{aligned}
$$

It is clear from (14) that the third solution depends on the arbitrary function $B$, i.e., we can generate the previous solutions of (12) and (13) by choosing the arbi- 
trary function $B$ to have the form

$$
B(R)=1 \quad \text { and } \quad B(R)=\int \frac{1}{R}\left(1-\sqrt{1-\frac{2 m}{R}-\frac{q^{2}}{R^{2}}}\right) \mathrm{d} R .
$$

The associated metric of the three solutions (12)-(14) is found to be the same and have the form

$$
\mathrm{d} s^{2}=-\eta(r) \mathrm{d} T^{2}+\frac{\mathrm{d} r}{\eta(r)}+r^{2} \mathrm{~d} \Omega^{2} \quad \text { with } \quad \eta(r)=1-\frac{2 m}{r}+\frac{q^{2}}{r^{2}},
$$

which is the static Reissner-Nordström black hole [26, 27]. The form of the vector potential $A_{\mu}$, the antisymmetric electromagnetic tensor field $F_{\mu \nu}$ and the energy-momentum tensor are given by [19]

$$
A_{t}(r)=-\frac{q}{2 \sqrt{\pi} r}, F_{r t}=-\frac{q}{2 \sqrt{\pi} r^{2}}, T_{0}{ }^{0}=T_{1}{ }^{1}=-T_{2}{ }^{2}=-T_{3}{ }^{3}=\frac{q^{2}}{8 \pi r^{4}} .
$$

It is of interest to note that the two tensors $H_{\mu \nu}$ and $K_{\mu \nu}$ are vanishing identically for the three solutions given by Eqs. (12), (13), and (14). It is proved that these tensors are vanishing identically for any spherically symmetric solutions [28, 29].

\section{Singularities}

In teleparallel theories we mean by singularity of space-time [25] the singularity of the scalar concomitants of the torsion and curvature tensors.

Using the definitions of the Riemann-Christoffel curvature tensor, Ricci tensor, Ricci scalar, torsion tensor, basic vector, traceless part, and the axial vector part [30] we obtain for the first solution of (12):

$$
\begin{aligned}
& R^{\mu \nu \lambda \sigma} R_{\mu \nu \lambda \sigma}=\frac{8}{R^{8}}\left(7 q^{4}-12 R M q^{2}+6 M^{2} R^{2}\right), \quad R^{\mu \nu} R_{\mu \nu}=\frac{4 q^{4}}{R^{8}}, \quad R=0 \\
& T^{\mu \nu \lambda} T_{\mu \nu \lambda}=\frac{-2}{R^{4}\left(R^{2}-2 M R+q^{2}\right)}\left(4 R^{4}-12 M R^{3}+6 R^{2} q^{2}\right. \\
& -4 R^{3} \sqrt{R^{2}-2 M R+q^{2}}-10 R M q^{2}+8 R^{2} \sqrt{R^{2}-2 M R+q^{2}} M \\
& \left.-4 R \sqrt{R^{2}-2 M R+q^{2}} q^{2}+9 M^{2} R^{2}+3 q^{4}\right) \\
& \Phi^{\mu} \Phi_{\mu}=\frac{-1}{R^{4}\left(R^{2}-2 M R+q^{2}\right)}\left(-2 R^{3}+4 M R^{2}-2 R q^{2}\right. \\
& +2 \sqrt{R^{2}-2 M R+q^{2}} R^{2}-3 \sqrt{R^{2}-2 M R+q^{2}} M R \\
& \left.+\sqrt{R^{2}-2 M R+q^{2}} q^{2}\right)^{2}, \\
& t^{\mu \nu \lambda} t_{\mu \nu \lambda}=\frac{-1}{R^{4}\left(R^{2}-2 M R+q^{2}\right)}\left(R^{3}-2 M R^{2}+R q^{2}\right. \\
& -\sqrt{R^{2}-2 M R+q^{2}} R^{2}+3 \sqrt{R^{2}-2 M R+q^{2}} M R \\
& \left.-2 \sqrt{R^{2}-2 M R+q^{2}} q^{2}\right)^{2}, \quad a^{\mu} a_{\mu}=0 .
\end{aligned}
$$

The scalars of the Riemann-curvature tensor, Ricci tensor, and Ricci scalar of the 
second solution (13) are the same as given by (18). This is a logic result since both solutions reproduce the same metric tensor and these scalars mainly depend on the metric tensor. The scalars of torsion tensor, basic vector, traceless part, and the axial vector part of the space-time given by solution (13) are given by

$$
\begin{aligned}
& T^{\mu \nu \lambda} T_{\mu \nu \lambda}=\frac{-2}{\left(2 M r-q^{2}\right) r^{4}}\left(3 q^{4}-10 q^{2} M r+9 M^{2} r^{2}\right), \\
& \Phi^{\mu} \Phi_{\mu}=\frac{-1}{\left(2 M r-q^{2}\right) r^{4}}\left(3 M r-q^{2}\right), \\
& t^{\mu \nu \lambda} t_{\mu \nu \lambda}=\frac{-1}{\left(2 M r-q^{2}\right) r^{4}}\left(3 M r-2 q^{2}\right) .
\end{aligned}
$$

It is clear from (18) and (19) that the scalars of the torsion, basic vector, and the traceless part of the first two solutions given by Eqs. (12) and (13) are quite different in spite of the fact that they gave the same associated metric (16)! The singularity of the scalars of Riemann-curvature tensor, Ricci tensor, and Ricci scalar is given at $R \rightarrow 0$ and this is well known from general relativity as we can see from Eq. (18) [1]. As the singularities of the scalars of the torsion, basic vector, and the traceless part of the first solution (12) are $r \rightarrow 0$ and $R^{2}-2 M r+q^{2} \rightarrow 0$, the second singularity may have the form $R \rightarrow M \pm \sqrt{M^{2}-q^{2}}$, which is the horizon of the static Reissner-Nordström black hole [31].

The singularities of the second solution (19) are given by $r \rightarrow 0$ and $q^{2} / 2 M$. Now we have two solutions reproducing the same metric but the singularity of their space-times are not coinciding. This is expected of course due to the following facts:

i) The energy content of these space-times are different [19].

ii) The time-space components of the tetrad fields $b_{0}{ }^{\alpha}, b_{\alpha}{ }^{0}$ go to zero as $1 / \sqrt{r}$ at infinity $[28,29]$.

iii) Also another interpretation, which may be taken into account as it is clear from Eqs. (18) and (19), is that the torsion tensor of these solutions is different. As we discussed in the introduction that the torsion plays the role of the force, therefore, we may interpret the different results of the two torsions given by Eqs. (18) and (19) due to the fact that the forces of the two solutions are different.

\section{The stability condition}

In the background of gravitational field the trajectories are represented by the geodesic equation

$$
\frac{\mathrm{d}^{2} x^{\lambda}}{\mathrm{d} s^{2}}+\left\{\begin{array}{l}
\lambda \nu \\
\mu \nu
\end{array} \frac{\mathrm{d} x^{\mu}}{\mathrm{d} s} \frac{\mathrm{d} x^{\nu}}{\mathrm{d} s}=0,\right.
$$

where $\mathrm{d} x^{\mu} / \mathrm{d} s$ is the velocity 4 -vector, $s$ is a parameter varying along the geodesic. It is well known that the perturbation of the geodesic will lead to deviation [1]:

$$
\frac{\mathrm{d}^{2} \zeta^{\lambda}}{\mathrm{d} s^{2}}+2\left\{\begin{array}{l}
\lambda \\
\mu \nu
\end{array}\right\} \frac{\mathrm{d} x^{\mu}}{\mathrm{d} s} \frac{\mathrm{d} \zeta^{\nu}}{\mathrm{d} s}+\left\{\begin{array}{l}
\lambda \\
\mu \nu
\end{array}\right\}_{, \rho} \frac{\mathrm{d} x^{\mu}}{\mathrm{d} s} \frac{\mathrm{d} x^{\nu}}{\mathrm{d} s} \zeta^{\rho}=0,
$$

where $\zeta^{\rho}$ is the deviation 4 -vector. 
Inserting Eqs. (20) and (21) into (16) we get for the geodesic equations

$$
\frac{\mathrm{d}^{2} t}{\mathrm{~d} s^{2}}=0, \quad \frac{1}{2} \eta^{\prime}(r)\left(\frac{\mathrm{d} t}{\mathrm{~d} s}\right)^{2}-r\left(\frac{\mathrm{d} \phi}{\mathrm{d} s}\right)^{2}=0, \quad \frac{\mathrm{d}^{2} \theta}{\mathrm{d} s^{2}}=0, \quad \frac{\mathrm{d}^{2} \phi}{\mathrm{d} s^{2}}=0,
$$

and for the geodesic deviation

$$
\begin{aligned}
& \frac{\mathrm{d}^{2} \zeta^{0}}{\mathrm{~d} s^{2}}+\frac{\eta^{\prime}(r)}{\eta(r)} \frac{\mathrm{d} t}{\mathrm{~d} s} \frac{\mathrm{d} \zeta^{1}}{\mathrm{~d} s}=0 \\
& \frac{\mathrm{d}^{2} \zeta^{1}}{\mathrm{~d} s^{2}}+\eta(r) \eta^{\prime}(r) \frac{\mathrm{d} t}{\mathrm{~d} s} \frac{\mathrm{d} \zeta^{0}}{\mathrm{~d} s}-2 r \eta(r) \frac{\mathrm{d} \phi}{\mathrm{d} s} \frac{\mathrm{d} \zeta^{3}}{\mathrm{~d} s} \\
& \quad+\left[\frac{1}{2}\left(\eta^{\prime 2}(r)+\eta(r) \eta^{\prime \prime}(r)\right)\left(\frac{\mathrm{d} t}{\mathrm{~d} s}\right)^{2}-\left(\eta(r)+r \eta^{\prime}(r)\right)\left(\frac{\mathrm{d} \phi}{\mathrm{d} s}\right)^{2}\right] \zeta^{1}=0 \\
& \frac{\mathrm{d}^{2} \zeta^{2}}{\mathrm{~d} s^{2}}+\left(\frac{\mathrm{d} \phi}{\mathrm{d} s}\right)^{2} \zeta^{2}=0, \quad \frac{\mathrm{d}^{2} \zeta^{3}}{\mathrm{~d} s^{2}}+\frac{2}{r} \frac{\mathrm{d} \phi}{\mathrm{d} s} \frac{\mathrm{d} \zeta^{1}}{\mathrm{~d} s}=0,
\end{aligned}
$$

where $\eta(r)$ is defined by (16), $\eta^{\prime}(r)=\mathrm{d} \eta(r) / \mathrm{d} r$ and we have to consider the circular orbit in the plane

$$
\theta=\frac{\pi}{2}, \quad \frac{\mathrm{d} \theta}{\mathrm{d} s}=0, \quad \frac{\mathrm{d} r}{\mathrm{~d} s}=0 .
$$

Using (24) in (16) we get

$$
\eta(r)\left(\frac{\mathrm{d} t}{\mathrm{~d} s}\right)^{2}-r^{2}\left(\frac{\mathrm{d} \phi}{\mathrm{d} s}\right)^{2}=1,
$$

from (25) and (22) we obtain

$$
\left(\frac{\mathrm{d} \phi}{\mathrm{d} s}\right)^{2}=\frac{\eta^{\prime}(r)}{r\left[2 \eta(r)-r \eta^{\prime}(r)\right]}, \quad\left(\frac{\mathrm{d} t}{\mathrm{~d} s}\right)^{2}=\frac{2}{2 \eta(r)-r \eta^{\prime}(r)} .
$$

The variable $s$ in (23) can be eliminated and we can rewrite it in the form

$$
\begin{aligned}
& \frac{\mathrm{d}^{2} \zeta^{0}}{\mathrm{~d} \phi^{2}}+\frac{\eta^{\prime}(r)}{\eta(r)} \frac{\mathrm{d} t}{\mathrm{~d} \phi} \frac{\mathrm{d} \zeta^{1}}{\mathrm{~d} \phi}=0 \\
& \frac{\mathrm{d}^{2} \zeta^{1}}{\mathrm{~d} \phi^{2}}+\eta(r) \eta^{\prime}(r) \frac{\mathrm{d} t}{\mathrm{~d} \phi} \frac{\mathrm{d} \zeta^{0}}{\mathrm{~d} \phi}-2 r \eta(r) \frac{\mathrm{d} \zeta^{3}}{\mathrm{~d} \phi} \\
& \quad+\left[\frac{1}{2}\left(\eta^{\prime 2}(r)+\eta(r) \eta^{\prime \prime}(r)\right)\left(\frac{\mathrm{d} t}{\mathrm{~d} \phi}\right)^{2}-\left(\eta(r)+r \eta^{\prime}(r)\right)\right] \zeta^{1}=0 \\
& \frac{\mathrm{d}^{2} \zeta^{2}}{\mathrm{~d} \phi^{2}}+\zeta^{2}=0, \quad \frac{\mathrm{d}^{2} \zeta^{3}}{\mathrm{~d} \phi^{2}}+\frac{2}{r} \frac{\mathrm{d} \zeta^{1}}{\mathrm{~d} \phi}=0 .
\end{aligned}
$$

It is clear from the third equation of (27) that it represents a simple harmonic motion, this means that the motion in the plane $\theta=\pi / 2$ is stable. 
Let us assume now that the solution of the remaining equations is given by

$$
\zeta^{0}=A_{1} \mathrm{e}^{\mathrm{i} \omega \phi}, \quad \zeta^{1}=A_{2} \mathrm{e}^{\mathrm{i} \omega \phi}, \quad \text { and } \quad \zeta^{3}=A_{3} \mathrm{e}^{\mathrm{i} \omega \phi},
$$

where $A_{1}, A_{2}$, and $A_{3}$ are constants to be determined. Inserting (28) into (27) we get

$$
\frac{r^{3} m-6 m^{2} r^{2}+9 m r q^{2}-4 q^{4}}{r^{2}\left(m r-q^{2}\right)}>0
$$

which is the condition of the stability for a static spherically symmetric ReissnerNordström solution. Condition (29) can be rewritten as

$$
r-\frac{q^{2}}{m}>0 \text { and } \quad r-6 m>0 .
$$

\section{Main results}

The main results can be summarized as follows:

1) The singularity problem of the first two solutions (12) and (13) obtained before [19] has been studied. The scalars of the torsion tensor, basic vector, and the traceless part of these solutions are quite different as we can see from Eqs. (18) and (19). The scalars have a common singularity if $r \rightarrow 0$. Furthermore, the first solution has another singularity if

$$
r^{2}-2 r m+q^{2} \rightarrow 0,
$$

while the second solution has another singularity if

$$
r-\frac{q^{2}}{2 M} \rightarrow 0
$$

This explains that the structure of the two solutions (12) and (13) is quite different in spite of the fact that they reproduce the same metric space-time.

2) The stability condition for the metric of Reissner-Nordström black hole (Eq. (16)) is derived and given by Eq. (29). From this condition we can see that:

i) If $r \rightarrow 0$, the value of (29) is finite.

ii) If $r$ becomes large, then Eq. (30) takes the value $r>6 m$ and $r>q^{2} / m$, which is the condition of stability for Reissner-Nordström black hole.

iii) When $q=0$ and if $r$ becomes large, then Eq. (32) takes the value $r>6 \mathrm{~m}$, which is the condition of stability for the Schwarzschild black hole [30]. The analysis given here for the derivation of the stability condition is straightforward and simpler than that used in the literature [32, 33].

\section{References}

[1] R. d'Inverno, Introducing Einstein's Relativity, Oxford University Press, New York 1992.

[2] S.W. Hawking, G.F.R. Ellis, The Large Scale Structure of Spacetime, Cambridge University Press, London 1973.

[3] S.W. Hawking, W. Israel, An Einstein Centenary Survey, Cambridge University Press, Cambridge 1979. 
[4] S.W. Hawking, W. Israel, 300 Years of Gravitation, Cambridge University Press, Cambridge 1987.

[5] C. Pellegrini, J. Plebanski, Mat. Fys. Skr. Dan. Vid. Selsk. 2, no. 4, 1 (1963).

[6] F.W. Hehl, J. Nitsch, P. von der Heyde, in: General Relativity and Gravitation, Ed. A. Held, Plenum Press, New York 1980.

[7] K. Hayashi, T. Nakano, Prog. Theor. Phys. 38, 491 (1967).

[8] W. Kopczyński, J. Phys. A 15, 493 (1982).

[9] J.M. Nester, Class. Quantum Grav. 5, 1003 (1988).

[10] N. Toma, Prog. Theor. Phys. 86, 659 (1991).

[11] T. Kawai, N. Toma, Prog. Theor. Phys. 87, 583 (1992).

[12] V.C. de Andrade, J.G. Pereira, Phys. Rev. D 56, 4689 (1997).

[13] V.C. de Andrade, L.C.T. Guillen, J.G. Pereira, Phys. Rev. Lett. 84, 4533 (2000).

[14] V.C. de Andrade, L.C.T. Guillen, J.G. Pereira, Phys. Rev. D 64, 027502 (2001).

[15] C. Møller, Mat. Fys. Medd. Dan. Vid. Selsk. 39, no. 13, 1 (1978).

[16] C. Møller, in: Proc. Int. School of Physics "Enrico Fermi", Ed. C. Møller, Academic Press, London 1962.

[17] J.W. Maluf, J. Math. Phys. 35, 335 (1994).

[18] J.W. Maluf, J.F. DaRocha-neto, T.M.L. Toribio, K.H. Castello-Branco, Phys. Rev. D 65, 124001 (2002).

[19] Gamal G.L. Nashed, Mod. Phys. Lett. A 21, 2241 (2006).

[20] H.P. Robertson, Ann. Math. (Princeton) 33, 496 (1932).

[21] M.I. Wanas, M.A. Bakry, to be published.

[22] K. Hayashi, T. Shirafuji, Phys. Rev. D 19, 3524 (1979).

[23] E. Cartan, C.R. Acad. Sci. 174, 734, 593 (1922).

[24] F.I. Mikhail, M.I. Wanas, A. Hindawi, E.I. Lashin, Int. J. Theor. Phys. 32, 1627 (1993).

[25] T. Kawai, N. Toma, Prog. Theor. Phys. 83, 1 (1990).

[26] D. Garfinkle, G.T. Horowitz, A. Strominger, Phys. Rev. D 43, 3140 (1991).

[27] N. Bretón, gr-qc/0109022.

[28] T. Shirafuji, G.G.L. Nashed, K. Hayashi, Prog. Theor. Phys. 95, 665 (1996).

[29] G.G.L. Nashed, Nuovo Cimento B 117, 521 (2002).

[30] G.G.L. Nashed, Chaos, Solitons and Fractals 15, 841 (2003).

[31] K. Hong, E. Teo, Class. Quantum Grav. 20, 3269 (2003).

[32] S.I. Vacaru, Int. J. Mod. Phys. D 12, 461 (2003).

[33] R.S. Ward, Class. Quantum Grav. 19, L17 (2002). 